\title{
Uncertainty and Stepwise Investment*
}

\author{
Peter M. Kort $†$ Pauli Murto $\ddagger$ and Grzegorz Pawlina ${ }^{\S}$
}

\begin{abstract}
We analyze the optimal investment strategy of a firm that can complete a project either in one stage at a single freely chosen time point or in incremental steps at distinct time points. The presence of economies of scale gives rise to the following trade-off: lumpy investment has a lower total cost, but stepwise investment gives more flexibility by letting the firm choose the timing individually for each stage. Our main question is how uncertainty in market development affects this tradeoff. The answer is unambiguous and in contrast with a conventional real-options intuition: higher uncertainty makes the single-stage investment more attractive relative to the more flexible stepwise investment strategy.
\end{abstract}

Keywords: Investment analysis, real options, capital budgeting, project flexibility, dynamic programming

*We thank two anonymous referees, Engelbert Dockner, David Hobson, Ashay Kadam, participants of the Annual International Real Options Conference (Montréal), EFA (Ljubljana), EFMA (Milan), and seminar participants at Antwerp, Cass (London), Exeter, Tilburg, Warsaw and Warwick for helpful comments and suggestions. All remaining errors are ours.

${ }^{\dagger}$ Corresponding author. Department of Econometrics \& Operations Research and CentER, and Tilburg University, P.O. Box 90153, 5000 LE Tilburg, The Netherlands and Department of Economics, University of Antwerp, Prinsstraat 13, 2000 Antwerp 1, Belgium. Phone: +31 13 4662062, fax: +31 13 4663280, email: kort@uvt.nl

${ }^{\ddagger}$ Department of Economics, Helsinki School of Economics, P.O. Box 1210, FIN-00101, Finland

${ }^{\S}$ Department of Accounting and Finance, Lancaster University, Lancaster, LA1 4YX, UK 


\section{Introduction}

The relationship between economic uncertainty and investment is one of the central issues in modern capital budgeting, as reflected in the theory of real options (see, e.g., d'Halluin et al. (2007), Clark and Easaw (2007), Fontes (2008), Bøckman et al. (2008), and De Reyck et al. (2008)). The literature on real options emphasizes various forms of managerial flexibility in this context (see, e.g., Triantis and Hodder (1990) and Trigeorgis (1996)). On the basis of that literature, we are used to thinking that the value of flexibility is positively related to the level of uncertainty. For example, the notion of a positive value of flexibility in an uncertain environment is presented in Chapter 1 of Trigeorgis (1996): "As new information arrives and uncertainty about market conditions and future cash flows is gradually resolved, management may have valuable flexibility to alter its initial operating strategy in order to capitalize on favorable opportunities."

In this paper we consider a particular (but common) form of flexibility and provide a result that calls for a refinement of this view. We analyze a continuous-time model of investment that follows closely the standard real-options framework. As in McDonald and Siegel (1986), a firm can choose the optimal time to invest in an irreversible project whose present value depends on the stochastic market environment. The difference with the standard model is that, besides the possibility of undertaking the whole project at a single, freely chosen point in time, the firm can also complete it through incremental steps. We assume that there are scale economies that give rise to the following tradeoff: completing the investment in a single stage saves on total costs, while proceeding stepwise gives additional flexibility as the firm can respond to resolving uncertainty by choosing the investment timing individually for each step.

Concerning the effect of uncertainty on this trade-off, the basic real-options intuition seems to suggest that uncertainty favors flexibility at the expense of scale economies. Dixit and Pindyck (1994) devote section 2.5 of their book to this issue and indicate that it is uncertainty that makes flexibility relevant in the first place: "When the growth of demand is uncertain, there is a trade-off between scale economies and the flexibility that is gained by investing more frequently in small increments to capacity as they are needed". Yet our main result is the opposite: the higher the level of uncertainty, the more attractive the lumpy investment strategy relative to the stepwise investment. In this context, therefore, the payoff of the project being uncertain actually favors scale economies at the expense of flexibility. 


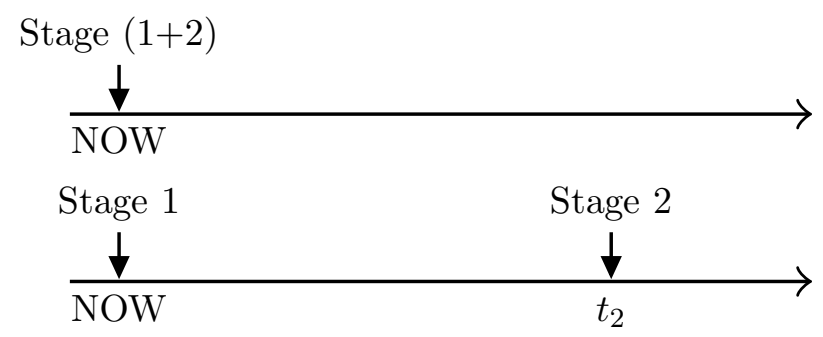

Panel B

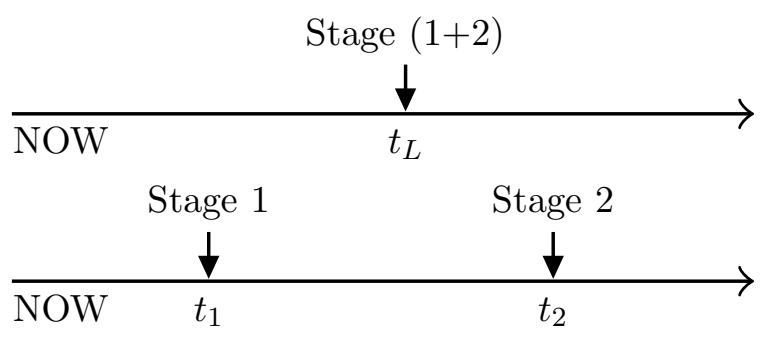

Figure 1: The timeline of the lumpy and stepwise investments (the top and the bottom axis in each pair, respectively) with a commitment to undertake stage 1 immediately (Panel A) and without any constraints on the timing of stage 1 (Panel B).

To understand this seemingly paradoxical result, it is necessary to look beyond a superficial real-options intuition and to carefully consider what is meant by flexibility in different contexts. The classical result is that the level of uncertainty is positively related to the value of an option to undertake an irreversible investment. One may express this by saying that the option to invest is a flexible asset, and the value of this flexibility increases with uncertainty (option to invest has a positive vega ${ }^{1}$ ). In this statement, flexibility refers to the presence of an option to choose the investment timing freely as opposed to a commitment to undertake a particular course of action at a given moment. Similarly, consider the choice between two investment strategies illustrated in Figure 1 (Panel A): either invest in the whole project now (lumpy investment) or

\footnotetext{
${ }^{1}$ Recall that an option vega is the first-order derivative of the option value with respect to the volatility of the underlying asset and is positive in a typical case.
} 
complete a fraction of the project now and keep an option to finish the remaining fraction later (a flexible alternative). Here flexibility stands for the increased degree of freedom in adding capacity, when the firm is allowed to do this in two steps rather than in one lump. We study the relative value of the two investment strategies in case the project has an uncertain payoff. This uncertainty can be caused, for instance, by (future) demand being stochastic. Again, higher uncertainty increases the relative value of flexibility via its impact on the value of the second-stage option. However, this comparison rests crucially upon the fact that the lumpy investment faces a restriction on its timing (the implicit now-or-never assumption) and, by construction, cannot benefit from a higher payoff volatility.

In contrast, the model presented in this paper allows for an optimal timing decision for both steps of the flexible project without placing any constraints on the timing of the lumpy project either, as illustrated in Figure 1 (Panel B). So, in this paper flexibility refers to the possibility of choosing the timing of each step individually as opposed to the more restrictive case where a single timing decision must apply for the entire project. This differs from a "standard" notion of flexibility that refers to the possibility to choose the timing for a project (or a part of a project) freely as opposed to the commitment to invest immediately.

Why is it then that the value of flexibility reduces with uncertainty in our setting? We suggest two intuitions. The first one follows from the theory of option pricing. Both investment opportunities, the lumpy and the stepwise one, can be viewed as perpetual American options to acquire the same underlying asset (the final full-scale project). When uncertainty increases, the values of both options converge towards the value of the underlying asset. Consequently, the advantage of being able to choose the timing of each stage separately instead of choosing the timing for both stages jointly diminishes with uncertainty.

The second intuition builds on the well known insight of real-options theory according to which increased uncertainty results in more inertia, that is, a decision maker is more reluctant to make costly switches between states as a response to changes in the underlying stochastic variable (cf. the hysteresis effect in the entry-exit model of Dixit (1989)). Our problem can be viewed as a special kind of a capacity switching problem. Stepwise investment strategy contains two switches: the first switch increases capacity from zero level to intermediate level, and a later switch from intermediate to the final level. Lumpy investment strategy, on the other hand, skips the intermediate level, and instead switches directly to the full capacity mode, albeit after a longer waiting time. 
As uncertainty is increased, the firm is less willing to incur switching costs, which favors the lumpy investment strategy.

While our model is stylized, the result relates to many potential applications. As an example, think of a firm that considers entry into a new market segment and faces the choice between two alternative strategies: either proceed in multiple stages (for example, starting with the most profitable geographical market), or wait until demand has grown enough to justify a single large-scale entry. A related example is the construction of production capacity: the choice may be between installing one big unit and installing a number of smaller units. Or, consider the adoption of a new technology: a firm can either start with an investment in an intermediate technology, which allows a subsequent implementation of the next-generation technology at a lower cost, or the firm may save on total costs by waiting and later "leap-frogging" directly to the next-generation technology. Our results indicate that, depending on the context, increased uncertainty favors large-scale entry, large production plants, and technology leapfrogging. ${ }^{2}$ Note also that instead of referring to one project to be undertaken in one or two steps, our framework may just as well be interpreted as two distinct projects either carried out separately or pooled together at a discounted total cost. A similar trade-off also appears in the purchase decision of a consumer, who may buy different goods separately each at its individually optimal time, or purchase them together at a discounted price. With that interpretation, our results indicate that increased uncertainty favors bundling of projects and purchases.

Our model is closely related to a number of earlier papers. It has been recognized elsewhere that the degree of uncertainty influences the firm's choice between alternative investment strategies. Dixit (1993) analyzes the choice between mutually exclusive projects of different sizes, and shows that increased uncertainty favors a larger project. Related results on the relationship between uncertainty and sizes of investment projects appear already in Manne (1961), and later in Capozza and Li (1994), Bar-Ilan and Strange (1999) and Dangl (1999). Décamps et al. (2006), on the other hand, complete the analysis of Dixit (1993) by considering the state-contingent investment policy over

\footnotetext{
${ }^{2}$ In a similar context, Grenadier and Weiss (1997) show in a model with sequential technological innovations that increased uncertainty favors waiting until the final technology is invented. The difference is that in their model uncertainty concerns the arrival time of an improved technology, whereas in our model it concerns the market environment. Consequently, in their model the improved technology is adopted at an exogenously determined moment, while in our model the timing is endogenously determined. It should also be noted that Grenadier and Weiss derive their result by numerical simulations, while our results are derived analytically.
} 
the entire state space. Their main finding is that the interaction between mutually exclusive options gives rise to a dichotomous option exercise region (a property that holds also in our model). While their research question is thus entirely different, the model they use is technically related to ours. ${ }^{3}$ Another literature stream analyzes investment strategies in a R\&D context (see, e.g., Childs et al. (1998), Weitzman et al. (1981), Roberts and Weitzman (1981)). That literature, like our paper, highlights the value of stepwise investment strategies, but its focus is on an entirely different mechanism than ours. In that context the main advantage of stepwise investment is the additional information that completing a step generates for choosing subsequent actions. In our model there is no learning; the advantage is generated by the improved flexibility to choose investment timings as a response to an exogenously changing environment.

The remainder of the paper is organized as follows. Section 2 describes the model, while Section 3 derives the optimal investment policy. Section 4 explains how the policy is affected by the key model parameters, Section 5 discusses some generalizations, and Section 6 concludes. All proofs are relegated to the Appendix.

\section{Model}

Our framework is based on the standard model of irreversible investment under uncertainty as presented in McDonald and Siegel (1986), and further analyzed in a large number of contributions (an extensive overview of this literature is provided in Dixit and Pindyck (1994) as well as in Brennan and Trigeorgis (2000)).

Consider a risk-neutral, all-equity financed firm of given size, which operates in continuous time with an infinite horizon and discounts its revenues with a constant rate $r$. (Instead of risk neutrality, we could assume that the payout from the project can be replicated by a portfolio of traded assets.) The firm earns initially no revenue, but has an opportunity to invest in a single capital budgeting project. This project can be accomplished either in one stage (which is referred to as lumpy investment) or in two separate stages (stepwise investment). The timing of the project and the type of investment (lumpy vs. stepwise) is to be chosen optimally in order to maximize the value of the firm.

\footnotetext{
${ }^{3}$ The difference is that in their model two projects of different scale are mutually exclusive: the stepwise investment strategy requires full replacement of the small plant by the large plant. Our model allows one to proceed to the final "large" project either through one lump or through incremental steps with an arbitrary relationship between investment costs of various alternatives.
} 
Denote by $t \in[0, \infty)$ the time index. The uncertain payoff of the project is characterized by state variable $Y_{t}$ that follows a geometric Brownian motion:

$$
d Y_{t}=\mu Y_{t} d t+\sigma Y_{t} d \omega_{t}
$$

where $Y_{0}>0, \mu<r, \sigma>0$, and the $d \omega$ 's are independently and identically distributed according to a normal distribution with mean zero and variance $d t$.

We assume that the initial value $Y_{0}$ is so low that at time $t=0$ it is not yet optimal for the firm to undertake the project (in neither lumpy nor stepwise fashion). However, since from (1) we learn that $Y$ is fluctuating over time, with positive probability it will be optimal for this firm to invest at a later point of time. The fact that the firm will not invest at time zero will allow us to make our comparisons between various investment strategies simply on the basis of their respective value functions calculated at $Y_{0}$. We think that the assumption of a low initial value for $Y$ is very natural in the current context: to analyze how uncertainty affects the choice between various investment strategies, we want to model the conditions under which the investment becomes optimal for the first time. If the initial value were higher than that, there should be some reason for why the project has not yet been implemented before the "initial" time. We recognize, however, that analyzing the whole investment policy valid for arbitrary initial states would bring in additional aspects (see Décamps et al. (2006)).

The firm's revenues are modeled as follows. Initially, the firm generates no revenues. Once $k \in\{1,2\}$ stages of the project are completed, the firm earns an instantaneous revenue flow:

$$
\pi_{t}=Y_{t} \sum_{i=1}^{k} R_{i},
$$

where $R_{i}$ is a constant denoting the deterministic part of the revenue increment corresponding to stage $i$. Define $R \equiv R_{1}+R_{2}$. By accomplishing the project in a single step, the firm moves at some stopping time $t^{L}$ directly from revenue flow 0 to $Y_{t^{L}} R$ (lumpy investment), while by splitting the project, the firm moves first at some stopping time $t^{1}$ from 0 to $Y_{t^{1}} R_{1}$, and at a later stopping time $t^{2}$ from $Y_{t^{2}} R_{1}$ to $Y_{t^{2}} R$ (stepwise investment). The cost of investment is deterministic and depends on whether the project is accomplished in one or two steps. In case of lumpy investment, the total investment cost is simply $I$. In case of stepwise investment, the associated investment costs for the first and second steps are $I_{1}(<I)$ and $I_{2}$, respectively. The firm's optimal strategy can thus be characterized as the following maximization problem (recall that $Y_{0}$ is so low that it is not optimal to invest at $t=0$ ): 


$$
\begin{aligned}
F\left(Y_{0}\right)= & \max \left\{F_{L}\left(Y_{0}\right) ; F_{S}\left(Y_{0}\right)\right\}=\max \left\{\sup _{t^{L} \geq 0} \mathbb{E}\left(\int_{t^{L}}^{\infty} e^{-r t} Y_{t} R d t-e^{-r t^{L}} I\right) ;\right. \\
& \left.\sup _{t^{1} \geq 0}\left(\sup _{t^{2} \geq t^{1}} \mathbb{E}\left(\int_{t^{1}}^{t^{2}} e^{-r t} Y_{t} R_{1} d t-e^{-r t^{1}} I_{1}+\int_{t^{2}}^{\infty} e^{-r t} Y_{t} R d t-e^{-r t^{2}} I_{2}\right)\right)\right\},
\end{aligned}
$$

where $t^{L}, t^{1}$, and $t^{2}$ are stopping times adapted to $Y_{t}$. The first term in the brackets, $F_{L}\left(Y_{0}\right)$, is the expectation of the discounted future revenues if the lumpy investment is chosen. Here, the firm chooses the stopping time $t^{L}$ at which the project is undertaken. The second term, $F_{S}\left(Y_{0}\right)$, corresponds to the stepwise investment. Then, the firm chooses two stopping times, $t^{1}$ and $t^{2}$, corresponding to stages 1 and 2 of the project, respectively. Whether the firm chooses the lumpy or the stepwise alternative depends on which of the two terms is greater.

We adopt the following assumptions on the investment costs and revenues. First, we assume that completing the project in two steps is more costly than investing in one lump and define

$$
\kappa \equiv\left(I_{1}+I_{2}\right) / I \geq 1
$$

Consequently, $\kappa$ represents the premium for flexibility that the firm must pay in order to be able to split the project. Second, without loss of generality, we assume that

$$
\frac{I_{1}}{I_{2}}<\frac{R_{1}}{R_{2}}
$$

As it becomes clear later, this implies that even if we interpret the model so that the firm is free to choose the order in which the two steps are undertaken, the step that will be optimally completed first is the one labelled with subscript 1 . We only assume away the trivial case $\frac{R_{1}}{R_{2}}=\frac{I_{1}}{I_{2}}$, which would imply that it is always optimal to undertake the two steps at the same point of time. In that case the firm does not benefit from the possibility to split the project, and the lumpy project with no cost premium would always dominate. (The latter conclusion would also hold if the firm were not able to first invest in the more profitable stage of project due to, for example, technological constraints.) Our aim is thus to focus on the effect of uncertainty on the trade-off between economics of scale and flexibility. To keep the analysis as simple as possible, we abstract from incorporating elements like operating costs, although we are well aware that operating costs can influence this trade-off. This is because a single stage investment will put much stress on the organization with increased operating costs as 
a result. On the other hand, investing stepwise will allow for gradual adaptation of routines and a gradual build up of internal competence.

To facilitate the communication of our results, we divide the parameters of the model into two classes. First, parameters $\mu, \sigma$, and $r$ describe the economic environment in which the firm operates, and we call them the market-specific parameters. Second, the parameters $R_{1}, R_{2}, I_{1}, I_{2}$, and $I$ describe the project under consideration, and we call them the project-specific parameters. Our purpose is to show how changes in market-specific parameters affect the regions in the space of project specific parameters in which each of the two alternative investment strategies (lumpy vs. stepwise) dominates. This approach will provide an unambiguous answer to our main question, that is, how the degree of uncertainty affects the optimal choice between the stepwise and lumpy investment and, hence, the relative value of flexibility.

\section{Optimal Investment Policy}

In this section, we derive the optimal solution to the project selection problem (3) in three steps. First, we consider the case where only the lumpy investment alternative is available. Second, we derive the optimal investment policy for the stepwise investment case. Finally, we consider the general problem where the firm has to decide about both the timing and the type of investment.

\subsection{Lumpy investment}

Consider the case in which the project can be undertaken in a single step only. Then the value of the investment opportunity is the first term between the brackets in (3), that is, the problem is to choose $t^{L}$ optimally to yield the value $F_{L}\left(Y_{0}\right)$ :

$$
F_{L}\left(Y_{0}\right)=\sup _{t^{L} \geq 0} \mathbb{E}\left(\int_{t^{L}}^{\infty} e^{-r t} Y_{t} R d t-e^{-r t^{L}} I\right) .
$$

This case corresponds exactly to the basic model of investment under uncertainty as described in McDonald and Siegel (1986). The optimal investment policy is a trigger strategy such that it is optimal to invest whenever the current value of $Y$ is above a certain threshold level, which we denote by $Y_{L}$. Thus, the optimal investment time is $t^{L}=\inf \left\{t \geq 0 \mid Y_{t} \geq Y_{L}\right\}$. The standard procedure to solve the problem is to set up the dynamic programming equation for the value function $F_{L}\left(Y_{0}\right)$, where the application 
of Itô's lemma and appropriate boundary conditions are used to determine the exact form of $F_{L}\left(Y_{0}\right)$ and the value of $Y_{L}$. We merely state the result here, see e.g. Dixit and Pindyck (1994) for details. The investment threshold is

$$
Y_{L}=\frac{\beta}{\beta-1} \frac{I}{R}(r-\mu)
$$

where

$$
\beta=\frac{1}{2}-\frac{\mu}{\sigma^{2}}+\sqrt{\left(\frac{1}{2}-\frac{\mu}{\sigma^{2}}\right)^{2}+\frac{2 r}{\sigma^{2}}}>1
$$

and the value of the option to invest is

$$
F_{L}\left(Y_{0}\right)=\left(\frac{Y_{L} R}{r-\mu}-I\right)\left(\frac{Y_{0}}{Y_{L}}\right)^{\beta} .
$$

Expression (8) is valid when initial demand is so low that it is not optimal to invest right away, that is when $Y_{0}<Y_{L}$.

\subsection{Stepwise investment}

Now, consider the case in which the firm splits the project into two stages. Then the value of the investment opportunity is the second term between the brackets in (3), that is, the problem is to choose $t^{1}$ and $t^{2}$ optimally to yield the value $F_{S}\left(Y_{0}\right)$ :

$$
F_{S}\left(Y_{0}\right)=\sup _{t^{1} \geq 0} \mathbb{E}\left(\sup _{t^{2} \geq t^{1}} \mathbb{E}\left(\int_{t^{1}}^{t^{2}} e^{-r t} R_{1} d t-e^{-r t^{1}} I_{1}+\int_{t^{2}}^{\infty} e^{-r t} R d t-e^{-r t^{2}} I_{2}\right)\right) .
$$

The option to invest in the first stage may be seen as a compound option, since accomplishing it generates an option to proceed to the next stage. ${ }^{4}$ However, since the instantaneous revenue (2) is additive in the revenue flows associated with each stage, the problem can be represented as two separate investment problems. This can be seen by re-writing (9) as

$$
F_{S}\left(Y_{0}\right)=\sup _{t^{1} \geq 0} \mathbb{E}\left(\int_{t^{1}}^{\infty} e^{-r t} R_{1} d t-e^{-r t^{1}} I_{1}\right)+\sup _{t^{2} \geq t^{1}} \mathbb{E}\left(\int_{t^{2}}^{\infty} e^{-r t} R_{2} d t-e^{-r t^{2}} I_{2}\right)
$$

Expression (10) implies that the problem is decomposed into two stopping problems, which are only linked through the constraint $t^{2} \geq t^{1}$. For the moment, ignore this

\footnotetext{
${ }^{4}$ See Bar-Ilan and Strange (1998) for a more complex model of sequential investment that incorporates investment lags.
} 
constraint, and note that each of the two resulting problems is identical to the one considered in Section 3.1. Therefore, without constraint $t^{2} \geq t^{1}$, the solution must consist of two investment thresholds, $Y_{1}$ and $Y_{2}$, given by

$$
\begin{aligned}
Y_{1} & =\frac{\beta}{\beta-1} \frac{I_{1}}{R_{1}}(r-\mu), \\
Y_{2} & =\frac{\beta}{\beta-1} \frac{I_{2}}{R_{2}}(r-\mu) .
\end{aligned}
$$

Comparing these expressions, one can see immediately that $Y_{1}<Y_{2}$ under our assumption that $\frac{R_{1}}{R_{2}}>\frac{I_{1}}{I_{2}}$. Therefore, concerning the corresponding stopping times $t^{i}=\inf \left\{t \geq 0 \mid Y_{t} \geq Y_{i}\right\}, i \in\{1,2\}$, it must hold that $t^{2}>t^{1}$, which means that the constraint $t^{2} \geq t^{1}$ is automatically satisfied. We conclude that the first stage is accomplished strictly earlier than the second stage, and that the existence of stage 2 has no effect on the optimal exercise time of stage 1 , meaning that the two stages can be considered separately. ${ }^{5}$ We denote the values of the options to invest separately for the two stages as $F_{1}\left(Y_{0}\right)$ and $F_{2}\left(Y_{0}\right)$. Analogously to $(8)$, these can be written as

$$
\begin{aligned}
& F_{1}(Y)=\left(\frac{Y_{1} R_{1}}{r-\mu}-I_{1}\right)\left(\frac{Y_{0}}{Y_{1}}\right)^{\beta} \\
& F_{2}(Y)=\left(\frac{Y_{2} R_{2}}{r-\mu}-I_{2}\right)\left(\frac{Y_{0}}{Y_{2}}\right)^{\beta}
\end{aligned}
$$

and they are applicable for $Y_{0}<Y_{1}$ and $Y_{0}<Y_{2}$, respectively. The value of the (compound) option to invest sequentially can be written as:

$$
F_{S}\left(Y_{0}\right)=F_{1}\left(Y_{0}\right)+F_{2}\left(Y_{0}\right)=\left(\frac{Y_{1} R_{1}}{r-\mu}-I_{1}\right)\left(\frac{Y_{0}}{Y_{1}}\right)^{\beta}+\left(\frac{Y_{2} R_{2}}{r-\mu}-I_{2}\right)\left(\frac{Y_{0}}{Y_{2}}\right)^{\beta},
$$

which is again valid as long as $Y_{0}$ is low enough, in particular, when $Y_{0}<Y_{1}$.

\subsection{General problem}

So far, we have determined the option values and the optimal investment thresholds for the two investment strategies (lumpy and stepwise) separately. Now we consider the general problem (3). Since we have assumed that the initial value $Y_{0}$ is so low

\footnotetext{
${ }^{5}$ This result is due to the special structure of optimal stopping problems that also underlies the main conclusions of Leahy (1993) and Baldursson and Karatzas (1997), according to which an investor, who must take into account subsequent investments of the competitors, employs the same investment policy as a monopolist who is not threatened by such future events.
} 
that it is not optimal to undertake the project (in any mode) at $t=0$, the value of the firm is simply $F\left(Y_{0}\right)=\max \left\{F_{L}\left(Y_{0}\right) ; F_{S}\left(Y_{0}\right)\right\}$, where the expressions for $F_{L}\left(Y_{0}\right)$ and $F_{S}\left(Y_{0}\right)$ are given by (8) and (15), respectively. Our aim is to establish conditions that determine which of these expressions is greater. Since we are interested in the trade-off between the economies of scale and flexibility, we want to state the relation of the option values in terms of the parameter $\kappa$ that represents the cost premium that must be paid by the firm for the flexibility of splitting the investment.

The following proposition states that there is a single cut-off value such that if $\kappa$ is below that level, the option value corresponding to the stepwise investment strategy dominates that of lumpy investment, while the reverse is true for $\kappa$ above that level. Note that a similar interpretation of the domination relation of mutually exclusive options is implicitly adopted, for example, in Dixit (1993).

Proposition 1 Let $Y_{0}<Y_{1}$. There exists a critical level of the investment cost premium $\widehat{\kappa}>1$ such that when $\kappa=\widehat{\kappa}$, we have $F_{S}\left(Y_{0}\right)=F_{L}\left(Y_{0}\right)$, that is, the lumpy and stepwise investment strategies are equally good. The critical premium $\widehat{\kappa}$ can be expressed as follows:

$$
\widehat{\kappa}=\left[\gamma \Pi_{1}^{\beta-1}+(1-\gamma) \Pi_{2}^{\beta-1}\right]^{\frac{1}{\beta-1}}
$$

where

$$
\gamma \equiv \frac{R_{1}}{R}
$$

is the fraction of the payoff of the project generated after completing stage 1, and

$$
\Pi_{i} \equiv \frac{R_{i} / I_{i}}{R /\left(I_{1}+I_{2}\right)}
$$

is the relative profitability of (moneyness of the option associated with) stage $i$. For $\kappa<\widehat{\kappa}$, we have $F_{S}\left(Y_{0}\right)>F_{L}\left(Y_{0}\right)$, whereas for $\kappa>\widehat{\kappa}$, we have $F_{L}\left(Y_{0}\right)>F_{S}\left(Y_{0}\right)$.

Proof. See the Appendix.

Proposition 1 gives us an unambiguous dominance relation between the lumpy and stepwise investment. Note that $\widehat{\kappa}$ depends on the market-specific parameters $\mu, \sigma$, and $r$ through their effect on parameter $\beta$ only. Hence, $\beta$ aggregates the effect of the environment in which the firm operates on the choice between the lumpy and stepwise investment strategies.

The influence of the project-specific factors is captured by three parameters: the fraction of the total payoff from the project that can be attributed to the stage 1 investment, $\gamma$, and the profitability of each stage relative to the profitability of the 
project as a whole, $\Pi_{i}$. This profitability can also be directly interpreted as the relative moneyness of the option to invest in stage $i$.

\section{Effect of Uncertainty}

Our main objective is to show how the choice between lumpy and stepwise investment strategies depends on market-specific parameters. An increase (a decrease) in $\widehat{\kappa}$ is equivalent to a reduction (an expansion) of the set of project-specific parameter values under which the lumpy investment dominates (is dominated by) the stepwise investment. This leads to the interpretation that $\widehat{\kappa}$ represents the cost advantage of the lumpy investment required to compensate for the loss of flexibility in timing each step of the project separately. Thus, an increase (a decrease) in $\widehat{\kappa}$ is equivalent to a higher (lower) relative value of additional flexibility associated with stepwise investment.

From (16), we see that $\beta$ captures the effects of all market-specific parameters. The next proposition states our main result:

Proposition 2 Consider the critical cost premium $\widehat{\kappa}$ as a function of $\beta$. Then, the following relationship holds:

$$
\frac{\partial \widehat{\kappa}}{\partial \beta}>0
$$

This implies that the relative value of stepwise investment is negatively related to the volatility and to the drift rate of process (1), but positively related to the interest rate:

$$
\begin{aligned}
& \frac{\partial \widehat{\kappa}}{\partial \sigma}<0 \\
& \frac{\partial \widehat{\kappa}}{\partial \mu}<0 \\
& \frac{\partial \widehat{\kappa}}{\partial r}>0 .
\end{aligned}
$$

Proof. See the Appendix.

Equation (19) embodies the main result of this paper: increased uncertainty reduces the premium that the firm is willing to pay for the additional flexibility that the stepwise investment gives. The effects of the drift rate and interest rate, as given by (20) and (21) respectively, are less surprising, but they are nevertheless revealing. The intuition for $(20)$ is that if the rate at which the present value of the project grows is reduced $(\mu$ is reduced), the cost of delaying investment until it is optimal to undertake both stages 
together is increased, which makes the stepwise investment more attractive. This is quite obvious, but it completes our main argument: it is rather the fact that the growth is gradual (slow) that makes stepwise investment valuable in this context, not the fact that growth is uncertain. Of course, the effect of the interest rate, as expressed in (21), can be explained in a similar way: discounting revenues more heavily makes it more costly to delay investment until both stages are optimally undertaken together. Therefore, the relative value of stepwise investment is higher.

To understand the main result given in (19), it is helpful to use the following analogy. First, notice that (16) can be rearranged as:

$$
\widehat{\kappa}^{\beta-1}=\gamma \Pi_{1}^{\beta-1}+(1-\gamma) \Pi_{2}^{\beta-1}
$$

The left-hand side reflects the premium on the investment cost when selecting the stepwise investment, whereas the right-hand side is the benefit from timing the investment in each stage of the project optimally. The cutoff level of the premium, $\widehat{\kappa}$, can be therefore interpreted as the certainty equivalent of random payout $\Pi_{i}, i \in\{1,2\}$, (occurring with probability $\gamma$ and $1-\gamma$ ), when the utility function is of the form $u(x)=x^{\beta-1}$. It is straightforward to see that the certainty equivalent decreases with the concavity of the utility function. In the analyzed case, the latter is negatively related to $\beta$.

It is useful to think of our main result in yet another way. For $\kappa=1$ and finite $\sigma$, the value of the lumpy investment opportunity is always smaller than the value of the option to invest in a stepwise fashion (cf. (8) and (15)). When uncertainty tends to infinity, the values of both investment opportunities converge to the value of the underlying asset, that is, the present value of revenues generated in perpetuity by the full-scale project. (In fact, it is easy to show that the limit of (8) and (15) for $\sigma$ tending to infinity is equal to $Y R /(r-\mu)$.) Therefore, it is not surprising that for higher uncertainty the willingness to pay a premium for the ability to invest in a stepwise fashion is lower. (For a discussion of the limiting properties of $\widehat{\kappa}$ as volatility tends to infinity and zero, see the supplementary web appendix.)

Figure 2 provides a numerical illustration of the model. It depicts the relationship between the cut-off level of the flexibility premium, $\widehat{\kappa}$ and the volatility of the revenue flow, $\sigma$, with the following parameter values. The riskless interest rate is assumed to be $5 \%$ (which is approximately equal to the average yield on long-term treasury bonds based on the period 1926-99, as reported in Bodie et al. (2002)) and the drift rate of the project is assumed to be equal to $1.5 \%$ (implying a $3.5 \%$ return shortfall, which is approximately consistent with a long-term dividend yield as reported by Allen 


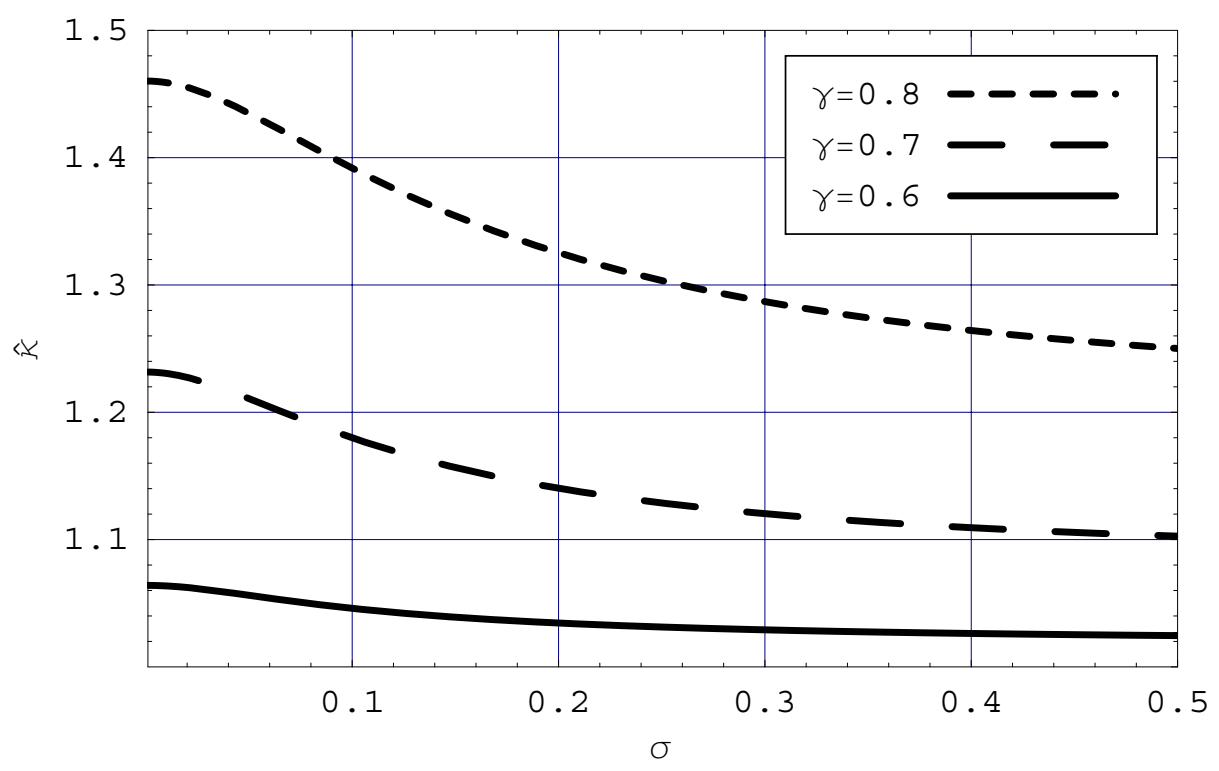

Figure 2: Flexibility premium cut-off level, $\widehat{\kappa}$, as a function of payoff volatility, $\sigma$, for different proportions of the payoff generated by stage $1, \gamma$. Other parameter values are as follows: $\alpha=0.5, r=0.05$, and $\mu=0.015$.

and Michaely (2002)). The proportion of the total investment cost incurred in each stage is one half $(\alpha=0.5)$. Consistent with the results of our model, we can see that the relationship between project flexibility (measured by $\widehat{\kappa}$ ) and uncertainty $(\sigma)$ is negative. Assuming that $I=\$ 100$, and that between three and four fifths of the revenue is generated following the completion of stage 1 , the maximum amount that the firm is willing to pay for flexibility ranges between $\$ 6$ and $\$ 46$ in the case of no uncertainty, and between $\$ 3$ and $\$ 32$ for $\sigma=0.2$ (the latter corresponds to the standard deviation of equity returns of a representative S\&P 500 firm, see Bodie et al. (2002)).

\section{$5 \quad$ Extensions}

Admittedly, the model is very simple and it is not obvious to what extent our main result is driven by the current specification of the project's payoff. For example, one might suspect that if, instead of a linear dependence like in our basic model, the project value is concave in the stochastic payoff $Y_{t}$, then the sign of the relationship between uncertainty and the value of flexibility will be reversed. This could be the case if the effect of a higher upside potential associated with a higher payoff volatility was dominated by its decreasing marginal contribution to the present value of the 
project. On the other hand, the notion of project flexibility is often associated with the possibility to revise investment decisions frequently (Trigeorgis (1996)), rather than merely adding a second step to an indivisible investment project. To deal with these issues, this section extends our result by considering a more general, power specification of the revenue function and by allowing for an arbitrary number of steps for which the timing can be independently chosen.

To account for a nonlinear impact of the market environment on the present value of the project, we allow the revenue flow to have a power specification (cf. (2)):

$$
\pi_{t}=\left(Y_{t} \sum_{i=1}^{n} R_{i}\right)^{\theta}, \theta \in(0, \beta)
$$

with $\theta<\beta$ required for obtaining finite valuations. For $\theta<1$, the firm is facing a concave revenue function, which may reflect, for example, constraints on input availability or, to some extent, imperfect hedging opportunities of risk averse shareholders. The case of $\theta>1$ may arise because of the firm's ability to respond to uncertainty, for example, by changing both price and quantity in response to demand fluctuations. In particular, one could think of a monopolist facing an isoelastic inverse demand function $p_{t}=Y_{t} q_{t}^{-1 / \theta}$ ( $p_{t}$ is price, $q_{t}$ is quantity). Assuming that the firm has constant marginal production $\operatorname{cost} c_{i}$, and no capacity constraint, the revenue flow with optimally chosen output level is given by $\pi_{t}=Y^{\theta} c_{i}^{1-\theta} \theta^{-1}\left(1-\frac{1}{\theta}\right)^{\theta-1}$, which is the same as (23) with an appropriately defined correspondence between $R_{i}$ and $c_{i}$. In that case, investment outlays should be interpreted as adoptions of different technologies that allow different marginal production $\operatorname{costs} c_{i}$.

Adopting the payoff function (23) with $n=2$ instead of (2) is in fact equivalent to transforming the stochastic process from $Y$ to $Y^{\theta}$ (with the relabeling of the parameters $R_{1}$ and $R_{2}$ as $R_{1}^{\prime} \equiv R_{1}^{\theta}$ and $R_{2}^{\prime} \equiv R^{\theta}-R_{1}^{\theta}$ ). Hence, the way to see that the results of Sections 3 and 4 generalize to the power function is to observe that $Y^{\theta}$ also follows a geometric Brownian motion but just with different drift and volatility terms than the original process $Y$. In fact, one can show (by applying Itô's lemma) that if $Y$ follows (1), the process $Y^{\theta}$ is a geometric Brownian motion with drift and volatility terms equal to $\mu^{\prime}=\theta \mu+\frac{1}{2} \theta(\theta-1) \sigma^{2}$ and $\sigma^{\prime}=\theta \sigma$, respectively. Hence, we may write $\beta^{\prime}=\frac{1}{2}-\frac{\mu^{\prime}}{\sigma^{\prime 2}}+\sqrt{\left(\frac{1}{2}-\frac{\mu^{\prime}}{\sigma^{\prime 2}}\right)^{2}+\frac{2 r}{\sigma^{\prime 2}}}=\frac{\beta}{\theta}$. The condition $\theta<\beta$ ensures that $\mu^{\prime}<r$, that is, that all the relevant valuations are finite.

In order to incorporate the notion that project flexibility is equivalent to the possibility of revising investment decisions frequently (Trigeorgis (1996)), we consider the 
optimal capital budgeting strategy when the firm is able to split the project into an arbitrary number of stages. The instantaneous revenue flow is now given by (23) with $n \geq 2$.

Define

$$
S_{k} \equiv \sum_{i=0}^{k} R_{i}
$$

for $k>0$, with $R_{0} \equiv 0$. In order to ensure that an investor chooses to accomplish stage $k$ at a strictly earlier time than stage $k+1$, we must have

$$
\frac{I_{k}}{I_{k+1}}<\frac{S_{k}^{\theta}-S_{k-1}^{\theta}}{S_{k+1}^{\theta}-S_{k}^{\theta}} \equiv \frac{R_{k}^{\prime}}{R_{k+1}^{\prime}}, \forall k<n .
$$

Now, it is possible to generalize the formula for the cut-off value of the flexibility premium (see the supplementary web appendix):

$$
\widehat{\kappa}=\left(\sum_{i=1}^{n} \gamma_{i}^{\prime} \Pi_{i}^{\prime \frac{\beta}{\theta}-1}\right)^{\frac{1}{\theta}-1},
$$

where $\gamma_{i}^{\prime} \equiv R_{i}^{\prime} / R^{\prime}$ and $\Pi^{\prime}$ is defined in the same way as $\Pi$ with $R\left(R_{i}\right)$ replaced by $R^{\prime}$ $\left(R_{i}^{\prime}\right)$. The proof that $\widehat{\kappa}$ increases with $\beta$, thus decreases with $\sigma$ is now analogous to the proof of Proposition 2.

To provide an illustration, Figure 3 depicts the cut-off level of the flexibility premium as a function of payoff convexity $\theta$ for different values of $n$. The cut-off level $\widehat{\kappa}$ decreases with the convexity of the revenue function. This implies that a firm holding a project with a more concave payoff function will, in fact, be willing to incur a higher additional costs to preserve flexibility in the investment process. The intuition for that result is that concavity increases the relative value of the initial stages of the project. This effect increases the value of the sequential strategy relative to the lumpy investment, simply because the stepwise strategy makes it possible to undertake the (more profitable) initial stages only. Furthermore, consistent with intuition, the value of the project flexibility is higher for a larger number of stages. For $I=\$ 100, \gamma_{i}=1.5 \gamma_{i+1}$ and $\alpha_{i}=1 / n$, the maximum level of flexibility premium ranges between $\$ 40$ and $\$ 117$ for a concave $(\theta=0.5), \$ 3$ and $\$ 17$ for a linear $(\theta=1)$, and between less than $\$ 1$ and $\$ 6$ for a convex payoff $(\theta=1.25)$.

\section{Conclusion}

We analyze the optimal investment strategy of a firm that can either accomplish the project in one lump or proceed in steps. The former strategy has the advantage of 


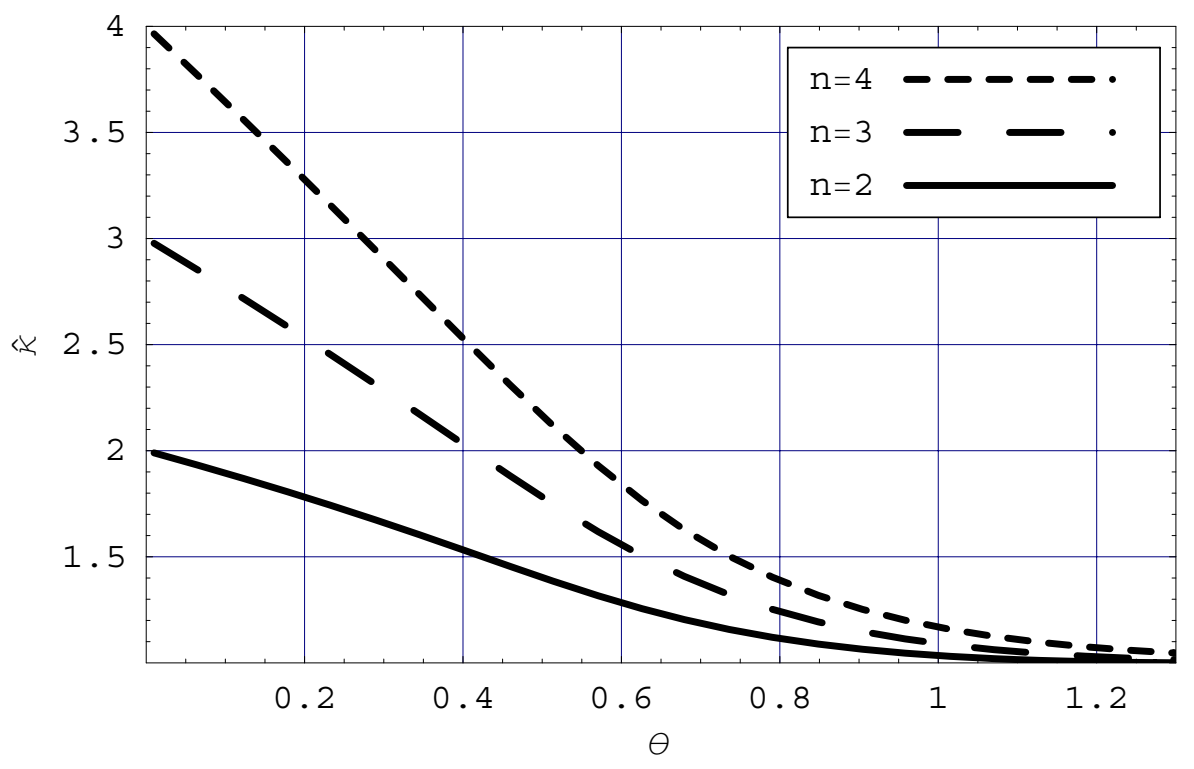

Figure 3: Flexibility premium cut-off level, $\widehat{\kappa}$, as a function of payoff convexity, $\theta$, for different numbers of stages, $n$. Other parameter values are as follows: $\alpha=0.5$, $\gamma_{i} / \gamma_{i+1}=1.5, r=0.05, \mu=0.015$, and $\sigma=0.2$.

scale economies: the total investment cost is lower. The latter benefits from additional flexibility: the timing of each step can be chosen independently of each other. Our focus is on the effect of uncertainty on the trade-off between the two strategies. The result is that increased uncertainty favors the lumpy investment relative to the stepwise investment.

One way to express our main result is to say that increased uncertainty reduces the value of flexibility associated with stepwise investment. This is in contrast with the standard real-options intuition, which says that increased uncertainty typically favors flexibility. Our key message is that such a statement - that is, uncertainty favors managerial flexibility - is not to be taken as a general fact. In particular, one must be careful in specifying what is meant by flexibility. If flexibility refers to the presence of an option to act later as opposed to the commitment to act now, then the standard result certainly holds: the value of flexibility increases with uncertainty. But if, as we show in this paper, flexibility refers to the possibility to split a project into a number of steps and to choose the timing of each step individually, then we have the opposite result: increased uncertainty reduces the value of flexibility. 


\section{A Appendix}

Proof of Proposition 1. We begin by comparing the two option values $F_{L}(Y)$ and $F_{S}(Y)$. It holds (cf. (8) and (15)) that

$$
\begin{aligned}
\frac{F_{S}(Y)}{F_{L}(Y)} & =\frac{\left(\frac{Y_{1} R_{1}}{r-\mu}-I_{1}\right)\left(\frac{Y}{Y_{1}}\right)^{\beta}+\left(\frac{Y_{2} R_{2}}{r-\mu}-I_{2}\right)\left(\frac{Y}{Y_{2}}\right)^{\beta}}{\left(\frac{Y_{L} R}{r-\mu}-I\right)\left(\frac{Y}{Y_{L}}\right)^{\beta}} \\
& =R^{-\beta}\left(\frac{I_{1}+I_{2}}{\kappa}\right)^{\beta-1}\left(\frac{R_{1}^{\beta}}{I_{1}^{\beta-1}}+\frac{R_{2}^{\beta}}{I_{2}^{\beta-1}}\right),
\end{aligned}
$$

since $I_{1}+I_{2} \equiv \kappa I$, and investment thresholds $Y_{L}, Y_{1}$, and $Y_{2}$, are given by (6), (11), and (12), respectively. Equation (16) follows directly from (A.1). Since $\beta$ is always greater than 1 , and all terms in (A.1) are positive, it holds that $\frac{\partial}{\partial \kappa}\left(\frac{F_{S}(Y)}{F_{L}(Y)}\right)<0$. This implies that $\frac{F_{S}(Y)}{F_{L}(Y)}=1$ if and only if $\kappa=\widehat{\kappa}$ and that the inequalities stated in the proposition hold.

Now, in order to prove that $\widehat{\kappa}>1$, we show that the $F_{S}(Y)>F_{L}(Y)$ for $\kappa=1$. Recall that $\alpha$ and $\gamma$ are defined (for an arbitrary $\kappa$ ) as

$$
\begin{aligned}
I_{1} & \equiv \alpha I \kappa, \\
R_{1} & \equiv \gamma R .
\end{aligned}
$$

Of course, (A.2) and (A.3) imply that $I_{2}=(1-\alpha) I \kappa$ and $R_{2}=(1-\gamma) R$. Define

$$
D(\beta) \equiv\left(\frac{\gamma^{\beta}}{\alpha^{\beta-1}}+\frac{(1-\gamma)^{\beta}}{(1-\alpha)^{\beta-1}}\right),
$$

which equals the ratio of $F_{S}(Y)$ and $F_{L}(Y)$ for $\kappa=1$. It can easily be seen that $\lim _{\beta \rightarrow 1}$ $D(\beta)=1$. To show that $D(\beta)>1$ for $\beta>1$, we calculate the following derivative

$$
\begin{aligned}
\frac{\partial D(\beta)}{\partial \beta} & =\frac{\partial}{\partial \beta}\left(\frac{\gamma^{\beta}}{\alpha^{\beta-1}}+\frac{(1-\gamma)^{\beta}}{(1-\alpha)^{\beta-1}}\right) \\
& =\gamma\left(\frac{\gamma}{\alpha}\right)^{\beta-1} \ln \frac{\gamma}{\alpha}+(1-\gamma)\left(\frac{1-\gamma}{1-\alpha}\right)^{\beta-1} \ln \frac{1-\gamma}{1-\alpha}
\end{aligned}
$$

For $\gamma \downarrow \alpha,(\mathrm{A} .5)$ is equal to zero. Therefore, in order to prove that (A.5) is positive, it is sufficient to show that

$$
\frac{\partial}{\partial \gamma}\left[\gamma\left(\frac{\gamma}{\alpha}\right)^{\beta-1} \ln \frac{\gamma}{\alpha}+(1-\gamma)\left(\frac{1-\gamma}{1-\alpha}\right)^{\beta-1} \ln \frac{1-\gamma}{1-\alpha}\right]
$$


is positive. Differentiating (A.6) and rearranging yields

$$
\left(\frac{\gamma}{\alpha}\right)^{\beta-1} \beta \ln \frac{\gamma}{\alpha}-\left(\frac{1-\gamma}{1-\alpha}\right)^{\beta-1} \beta \ln \frac{1-\gamma}{1-\alpha}+\left(\frac{\gamma}{\alpha}\right)^{\beta-1}-\left(\frac{1-\gamma}{1-\alpha}\right)^{\beta-1}>0
$$

The last inequality results from the fact that the first three components are positive and that $\frac{\gamma}{\alpha}>1>\frac{1-\gamma}{1-\alpha}$. Consequently, for $\kappa=1$ and $\beta>1$, the value of the sequential investment opportunity is higher than the value of the lumpy project. Since (A.1) decreases with $\kappa, \widehat{\kappa}$ is greater than 1 .

Proof of Proposition 2. $\widehat{\kappa}$ can be expressed as

$$
\widehat{\kappa}=\left(\gamma \Pi_{1}^{\beta-1}+(1-\gamma) \Pi_{2}^{\beta-1}\right)^{\frac{1}{\beta-1}} .
$$

Let us choose two arbitrary values of $\beta$, say $\beta^{\prime}$ and $\beta^{\prime \prime}$, such that $\beta^{\prime}>\beta^{\prime \prime}$, and define

$$
\delta \equiv \frac{\beta^{\prime}-1}{\beta^{\prime \prime}-1}>1
$$

It holds that

$$
\begin{aligned}
& \gamma \Pi_{1}^{\beta^{\prime \prime}-1}+(1-\gamma) \Pi_{2}^{\beta^{\prime \prime}-1}=\gamma \Pi_{1}^{\frac{\beta^{\prime}-1}{\delta}}+(1-\gamma) \Pi_{2}^{\frac{\beta^{\prime}-1}{\delta}} \\
< & \left(\gamma \Pi_{1}^{\beta^{\prime}-1}+(1-\gamma) \Pi_{2}^{\beta^{\prime}-1}\right)^{\frac{1}{\delta}}
\end{aligned}
$$

where the last inequality results from the fact that $y^{\frac{1}{\delta}}$ is a concave function. This implies the following inequality:

$$
\left(\gamma \Pi_{2}^{\beta^{\prime \prime}-1}+(1-\gamma) \Pi_{2}^{\beta^{\prime \prime}-1}\right)^{\delta}<\gamma \Pi_{1}^{\beta^{\prime}-1}+(1-\gamma) \Pi_{2}^{\beta^{\prime}-1}
$$

It follows immediately that

$$
\left(\gamma \Pi_{1}^{\beta^{\prime \prime}-1}+(1-\gamma) \Pi_{2}^{\beta^{\prime \prime}-1}\right)^{\frac{1}{\beta^{\prime \prime}-1}}<\left(\gamma \Pi_{1}^{\beta^{\prime}-1}+(1-\gamma) \Pi_{2}^{\beta^{\prime}-1}\right)^{\frac{1}{\beta^{\prime}-1}}
$$

Defining $\beta^{\prime} \equiv \beta^{\prime \prime}+\Delta \beta_{1}$ and letting $\Delta \beta$ tend to zero leads to the conclusion that $\partial \widehat{\kappa} / \partial \beta>0$. Results (19)-(21) follow from the fact that $\partial \beta / \partial \sigma<0, \partial \beta / \partial \mu<0$, and $\partial \beta / \partial r>0$, respectively. 


\section{References}

Allen, Franklin, and Roni Michaely, 2002, Payout policy, in George Constantinides, Milton Harris, and Rene Stulz, ed.: Handbook of the Economics of Finance (North Holland).

Baldursson, Fridrik, and Ioannis Karatzas, 1997, Irreversible investment and industry equilibrium, Finance and Stochastics 1, 69-89.

Bar-Ilan, Avner, and William C. Strange, 1998, A model of sequential investment, Journal of Economic Dynamics and Control 22, 437-463.

— 1999, The timing and intensity of investment, Journal of Macroeconomics 21, $57-78$.

Bøckman, Thor, Stein-Erik Fleten, Erik Juliussen, Håvard J. Langhammer, and Ingemar Revdal, 2008, Investment timing and optimal capacity choice for small hydropower projects, European Journal of Operational Research 190, 255-267.

Bodie, Zvi, Alex Kane, and Alan J. Marcus, 2002, Investments (McGraw-Hill Irwin) 5 edn.

Brennan, Michael J., and Lenos Trigeorgis (eds.), 2000, Project Flexibility, Agency and Competition (Oxford University Press).

Capozza, Dennis R., and Yuming Li, 1994, The intensity and timing of investment: The case of land, American Economic Review 84, 889-904.

Childs, Paul D, Steven H. Ott, and Alexander J. Triantis, 1998, Capital budgeting for interrelated projects: A real options approach, Journal of Financial and Quantitative Analysis 33, 305-334.

Clark, Ephraim, and Joshy Z. Easaw, 2007, Optimal access pricing for natural monopoly networks when costs are sunk and revenues are uncertain, European Journal of Operational Research 178, 595-602.

Dangl, Thomas, 1999, Investment and capacity choice under uncertain demand, European Journal of Operational Research 117, 1-14. 
De Reyck, Bert, Zeger Degraeve, and Roger Vandenborre, 2008, Project options valuation with net present value and decision tree analysis, European Journal of Operational Research 184, 341-355.

Décamps, Jean-Paul, Thomas Mariotti, and Stéphane Villeneuve, 2006, Irreversible investment in alternative projects, Economic Theory 28, 425-448.

d'Halluin, Yann, Peter. A. Forsyth, and Kenneth R. Vetzal, 2007, Wireless network capacity management: a real options approach, European Journal of Operational Research 176, 584-609.

Dixit, Avinash, 1989, Entry and exit decisions under uncertainty, Journal of Political Economy 97, 620-638.

— 1993 , Choosing among alternative discrete investment projects under uncertainty, Economics Letters 41, 265-268.

Dixit, Avinash K., and Robert S. Pindyck, 1994, Investment under Uncertainty (Priceton University Press).

Fontes, Dalila B. M. M., 2008, Fixed versus flexible production systems: A real options analysis, European Journal of Operational Research 188, 169-184.

Grenadier, Steven R., and Allen M. Weiss, 1997, Investment in technological innovations: An option pricing approach, Journal of Financial Economics 44, 397-416.

Leahy, John V., 1993, Investment in competitive equilibrium: The optimality of myopic behavior, Quarterly Journal of Economics 108, 1105-1133.

Manne, Alan S., 1961, Capacity expansion and probabilistic growth, Econometrica 29, $632-649$.

McDonald, Robert, and Daniel Siegel, 1986, The value of waiting to invest, Quarterly Journal of Economics 101, 707-728.

Roberts, Kevin, and Martin L. Weitzman, 1981, Funding criteria for research, development, and exploration projects, Econometrica 49, 1261-1288.

Triantis, Alexander J., and James E. Hodder, 1990, Valuing flexibility as a complex option, Journal of Finance 45, 549-565. 
Trigeorgis, Lenos, 1996, Real Options: Managerial Flexibility and Strategy in Resource Allocation (MIT Press).

Weitzman, Martin L., Whitney Newey, and Michael Rabin, 1981, Sequential R\&D strategy for synfuels, The Bell Journal of Economics 12, 574-590. 


\section{B Supplementary web appendix}

\section{Some limiting cases.}

To shed more light on the behavior of $\widehat{\kappa}$ as a function of volatility, we calculate its limits for $\sigma \rightarrow \infty$ and $\sigma \rightarrow 0$. It holds that (see below for the proofs)

$$
\lim _{\sigma \rightarrow \infty} \widehat{\kappa}=\Pi_{1}^{\gamma} \Pi_{2}^{1-\gamma}
$$

and

$$
\lim _{\sigma \rightarrow 0} \widehat{\kappa}= \begin{cases}\Pi_{1} & \mu \leq 0, \\ {\left[\gamma \Pi_{1}^{\frac{r-\mu}{\mu}}+(1-\gamma) \Pi_{2}^{\frac{r-\mu}{\mu}}\right]^{\frac{\mu}{r-\mu}}} & \mu>0 .\end{cases}
$$

In an extremely uncertain market $(\sigma \rightarrow \infty)$, the cutoff level of the flexibility premium is equal to the geometric weighted average of the relative profitabilities of the two stages of the project. Conversely, for a deterministic demand process $(\sigma \rightarrow 0)$, two cases are possible. If the drift rate $\mu$ of the demand process is non-positive, the cutoff level of the flexibility premium is equal to the maximum of the relative profitabilities of stages 1 and 2 (which is $\Pi_{1}$ by assumption (5)). For a strictly positive $\mu, \widehat{\kappa}$ is given by (16) where $\beta$ admits its limiting value for $\sigma \rightarrow 0$.

To interpret the meaning of the deterministic limit of $\widehat{\kappa}$ for $\mu \leq 0$, consider two cases - of a negative and of a zero NPV of the lumpy project. If the NPV of the whole (lumpy) project is negative (that is, if $Y R /(r-\mu)-I<0)$, then the firm will be indifferent between having the lumpy project and being able to invest sequentially with a flexibility premium equal to $\Pi_{1}$. In both cases, no investment will be made and the value of the firm is zero. In the special case of a zero NPV of the lumpy project, stepwise investment strictly dominates the lumpy one as long as $\kappa<\widehat{\kappa}=\Pi_{1}$. Then, the first step of the stepwise investment yields a strictly positive NPV and the second step is abandoned. The strictly positive sign of the first step is concluded by observing that

$$
N P V_{1}=\frac{Y R_{1}}{r-\mu}-I_{1}=\gamma\left(\frac{Y R}{r-\mu}-\frac{\kappa}{\Pi_{1}} I\right) .
$$

Obviously, this is greater than zero as long as $\kappa<\Pi_{1}$. 
Proof of (B.1) and (B.2). First, we prove (B.1).

$$
\begin{aligned}
\lim _{\sigma \rightarrow \infty} \widehat{\kappa} & =\lim _{\sigma \rightarrow \infty}\left[\gamma \Pi_{1}^{\beta-1}+(1-\gamma) \Pi_{2}^{\beta-1}\right]^{\frac{1}{\beta-1}} \\
& =\exp \left[\lim _{\sigma \rightarrow \infty} \frac{\log \left[\gamma \Pi_{1}^{\beta-1}+(1-\gamma) \Pi_{2}^{\beta-1}\right]}{\beta-1}\right] \\
& =\exp \left[\lim _{\sigma \rightarrow \infty} \frac{\gamma \Pi_{1}^{\beta-1} \log \Pi_{1}+(1-\gamma) \Pi_{2}^{\beta-1} \log \Pi_{2}}{\gamma \Pi_{1}^{\beta-1}+(1-\gamma) \Pi_{2}^{\beta-1}}\right] \\
& =e^{\gamma \log \Pi_{1}+(1-\gamma) \log \Pi_{2}} \\
& =\Pi_{1}^{\gamma} \Pi_{2}^{1-\gamma} .
\end{aligned}
$$

To prove (B.2), we first use the known property that $\lim _{\sigma \rightarrow 0} \beta$ equals infinity for $\mu \leq 0$ and $r / \mu$ otherwise (Dixit and Pindyck (1994)). Consequently, in the latter case the limit of $\widehat{\kappa}$ equals (16) with $\beta$ replaced by $r / \mu$. In the case of negative drift rate $\mu$, we use the three-series theorem (recall that $\Pi_{1}>\Pi_{2}$ ). It holds that

$$
\begin{aligned}
\gamma \Pi_{1}^{\beta-1}+(1-\gamma) \Pi_{2}^{\beta-1} & >\gamma \Pi_{1}^{\beta-1} \\
{\left[\gamma \Pi_{1}^{\beta-1}+(1-\gamma) \Pi_{2}^{\beta-1}\right]^{\frac{1}{\beta-1}} } & >\gamma^{\frac{1}{\beta-1}} \Pi_{1}
\end{aligned}
$$

and

$$
\begin{aligned}
\gamma \Pi_{1}^{\beta-1}+(1-\gamma) \Pi_{1}^{\beta-1} & >\gamma \Pi_{1}^{\beta-1}+(1-\gamma) \Pi_{2}^{\beta-1} \\
\Pi_{1} & >\left[\gamma \Pi_{1}^{\beta-1}+(1-\gamma) \Pi_{2}^{\beta-1}\right]^{\frac{1}{\beta-1}}
\end{aligned}
$$

Therefore

$$
\Pi_{1}>\left[\gamma \Pi_{1}^{\beta-1}+(1-\gamma) \Pi_{2}^{\beta-1}\right]^{\frac{1}{\beta-1}}>\gamma^{\frac{1}{\beta-1}} \Pi_{1}
$$

Since

$$
\lim _{\sigma \rightarrow 0} \gamma^{\frac{1}{\beta-1}} \Pi_{1}=\Pi_{1}
$$

it is also true that

$$
\lim _{\sigma \rightarrow 0} \widehat{\kappa}=\Pi_{1} .
$$

Investment thresholds and $\hat{\kappa}$ for general $\theta$.

With the payoff function (23), condition (5) is replaced by

$$
\frac{I_{1}}{I_{2}}<\frac{R_{1}^{\theta}}{R^{\theta}-R_{1}^{\theta}} \equiv \frac{R_{1}^{\prime}}{R_{2}^{\prime}},
$$


where we introduce the notation $R_{1}^{\prime}+R_{2}^{\prime} \equiv R^{\prime}=R^{\theta}$ (for $n>2$, (5) is replaced by (25)). This condition ensures that if the firm chooses the sequential investment strategy, the stage labeled with subscript 1 is optimally undertaken strictly before stage 2 . Before we proceed to analyzing the investment decision(s) of the firm, it is useful to establish the following present value:

$$
\mathbb{E}\left(\int_{t}^{\infty} e^{-r t} Y_{t}^{\theta} d t\right)=\frac{Y_{t}^{\theta}}{r-\theta \mu-\frac{1}{2} \theta(\theta-1) \sigma^{2}} \equiv \frac{Y_{t}^{\theta}}{\delta(\theta)} .
$$

Since for $x>0$ condition $\delta(x)>0$ is equivalent to $x<\beta$ (note that $\beta$, given by (7), is the positive root of the quadratic equation in the denominator of (B.7)), the convergence of the present value of the project revenues requires that $\theta<\beta$. Obviously, for linear and concave payoff functions this condition is always satisfied.

The option value of the project under the lumpy investment strategy is

$$
F_{L}(Y)=\sup _{t^{L} \geq 0} \mathbb{E}\left(\int_{t^{L}}^{\infty} e^{-r t} Y_{t}^{\theta} R^{\prime} d t-I e^{-r t^{L}}\right) .
$$

The resulting optimal investment threshold, $Y_{L}$, equals

$$
Y_{L}=\frac{1}{R}\left(\frac{\beta}{\beta-\theta} I \delta(\theta)\right)^{\frac{1}{\theta}}
$$

with the required mark-up on the investment cost now being equal to $\beta /(\beta-\theta)$, which increases with the payoff convexity. The value of the project can now be expressed as

$$
F_{L}(Y)=\left(\frac{Y_{L}^{\theta} R^{\prime}}{\delta(\theta)}-I\right)\left(\frac{Y}{Y_{L}}\right)^{\beta} .
$$

For $\theta=1,($ B.10) reduces to (8).

Analogously to (15), the value of the option to invest sequentially is

$$
F_{S}(Y)=F_{1}(Y)+F_{2}(Y)=\left(\frac{Y_{1}^{\theta} R_{1}^{\prime}}{\delta(\theta)}-I_{1}\right)\left(\frac{Y}{Y_{1}}\right)^{\beta}+\left(\frac{Y_{2}^{\theta} R_{2}^{\prime}}{\delta(\theta)}-I_{2}\right)\left(\frac{Y}{Y_{2}}\right)^{\beta}
$$

where

$$
Y_{i}=\left(\frac{\beta}{\beta-\theta} \frac{I_{i}}{R_{i}^{\prime}} \delta(\theta)\right)^{\frac{1}{\theta}},
$$

for $i \in\{1,2\}$ (for $n>2$, thresholds have an identical form). Now, we are able to derive the cut-off premium for flexibility

$$
\widehat{\kappa}=\left(\gamma^{\prime} \Pi_{1}^{\prime \frac{\beta}{\theta}-1}+\left(1-\gamma^{\prime}\right) \Pi_{2}^{\prime \frac{\beta}{\theta}-1}\right)^{\frac{1}{\theta}-1}
$$

where $\gamma^{\prime}$ and $\Pi_{i}^{\prime}$ are defined in an analogous way as in Proposition 1. 